\title{
Functional role of cationic surfactant to control the nano size of silica powder
}

\author{
L. P. Singh $\cdot$ S. K. Bhattacharyya $\cdot$ G. Mishra $\cdot$ \\ S. Ahalawat
}

Received: 18 April 2011 / Accepted: 11 July 2011 / Published online: 26 July 2011

(C) The Author(s) 2011. This article is published with open access at Springerlink.com

\begin{abstract}
Preparation of dispersed, amorphous, spherical silica nanoparticles using cationic surfactant as organic template, tetraethoxysilane (TEOS) as silica precursor and ammonia as catalyst has been carried out using sol gel process. The aim of the present study was to evaluate the simultaneous effects of cationic surfactant on the textural and structural properties of silica nanoparticles. We used a series of the cationic surfactants, dodecytrimethylammonium bromide (DTAB), tetradecyltrimethylammonium bromide (TTAB) and cetyltrimethylammonium bromide (CTAB) to evaluate the effects of the chain length of cationic surfactant on the grain size of silica nanoparticles. The size of silica nanoparticles can be finely tuned in the range $\sim 50-100 \mathrm{~nm}$ by changing the chain length of cationic surfactant. Decreasing the particle size of silica nano particles resulted in increase in chain length of cationic surfactant. Further, these silica nanoparticles are incorporated with cement paste to evaluate the beneficial effect on mechanical properties of cement. Synthesized silica nanoparticles were analyzed using scanning electron microscopy (SEM), ${ }^{29}$ Si MAS NMR, powder X-ray diffraction techniques (XRD) and IR studies.
\end{abstract}

Keywords Sol gel · Cationic surfactant . Silica nanoparticles $\cdot$ 29Si MAS-NMR .

Calcium leaching

L. P. Singh $(\bowtie) \cdot$ S. K. Bhattacharyya · G. Mishra .

S. Ahalawat

CSIR, Central Building Research Institute (CBRI),

Roorkee 247-667, Uttarakhand, India

e-mail: lpsingh@cbri.in

\section{Introduction}

Nanotechnology has traditionally contributed significantly to the development of the medical, biological, pharmaceutical, food, agricultural science and wide spectrum of engineering. It is a creation of functional materials, devices and systems through manipulation of matter in the nanometer scale and exploitation of novel phenomena and properties which arise because of the nanometer scale. Therefore, it is possible to design materials of required optical, magnetic, elastic and chemical properties by controlling the above factors.

Dispersed, amorphous and uniform silica nanoparticles have aroused specific interest due to their simple preparation and potential applications in various industries. It can be used as effective materials in various cementitious system for improving the strength, flexibility, durability, workability, etc. Various synthetic strategies have been reported to prepare silica nanoparticles, such as the modified Stöber method (Grün et al. 1999; Pauwels et al. 2001; Liu et al. 2003; Zhang et al. 2004; Lebedev et al. 2004; Tan et al. 2004; Shimura et al. 2005; Tan et al. 2005), combustion techniques (Hong et al. 2009), chemical vapor deposition (Awaji et al. 1997), aerosol spray (Rao et al. 2002; Bore et al. 2003; Miller et al. 2005), and emulsion (Oh et al. 2005) method. Various types of morphology such as hexagonal, cubic, lamellar, and wormhole like mesostructures have been synthesized using these methods. It is well-known that spherical dispersed silica nanoparticles can be prepared by using the sol gel method. The main advantage of this method is to control the particle size at nano level and morphology of silica nanoparticles by changing the concentration of reagents, type of catalyst, temperature, reaction conditions, etc. Many efforts have been made to control the particle size and morphology of 
silica nanoparticles using different type of surfactants as templates. Venkatathri has reported that mesoporous silica nanosphere was synthesized using polystyrene and cetyltrimethylammonium bromide as template (Venkatathri et al. 2009). Hu et al. have successfully synthesized silica hollow sphere of a high surface area with a diameter about $50 \mathrm{~nm}$ using $\mathrm{CaCO}_{3}$ as the template (Yongqi et al. 2008). Mesoporous silica nanospheres with uniform size and morphology have been synthesized using polyvinyl pyrrolidine and cetyltrimethylammonium bromide, cetyltrimethylammonium chloride and $n$-octylamine as template (Venkatathri 2007).

The application of nanomaterials in construction are expected to improve the essential properties of building materials such as strength, durability, etc., in novel collateral functions such as energy saving, self-healing, antifogging, etc., and to provide component for structuring health sensors. Presently, the focus of nanotechnology in construction is on cement-based materials and their fracture mechanism. Highly crystalline portlandite $(\mathrm{CH})$ and amorphous calcium-silicate-hydrate $(\mathrm{C}-\mathrm{S}-\mathrm{H})$ are the major phases formed during the hydration process. The main strength giving phase in cementitious system is Calcium-Silicate-Hydrate $(\mathrm{C}-\mathrm{S}-\mathrm{H})$ which is of the order of $1-100 \mathrm{~nm}$ range and size of pores present in $\mathrm{C}-\mathrm{S}-\mathrm{H}$ are also in nano to micrometer range. These pores with type and morphology of $\mathrm{C}-\mathrm{S}-\mathrm{H}$ gel control the properties of the hydration product. Leaching of calcium ions from cementitious matrices is a combined diffusion-dissolution process that results in long-term durability issues for cement-based materials.

In the preparation of silica nanoparticles, the rate and extent of the hydrolysis reaction of TEOS were found to be greatly influenced by reaction conditions, therefore, in the present study use of cationic surfactants has been explored to control the particle size of the silica at nano level and the effect of addition of these nanoparticles in cement pastes for mineralogical and morphological attributes were investigated through SEM and XRD analysis.

\section{Experimental}

Tetraethoxysilane (TEOS) (99.9\% Alfa Aesar, UK), absolute ethanol (99.9\% Merck, India), ammonia (25\% Thomas Baker, India) and dodecytrimethylammonium bromide $\left(\mathrm{C}_{15} \mathrm{H}_{34} \mathrm{BrN}\right)$, tetradecyltrimethylammonium bromide $\left(\mathrm{C}_{17} \mathrm{H}_{38} \mathrm{BrN}\right)$ and cetyltrimethylammonium bromide $\left(\mathrm{C}_{19} \mathrm{H}_{42} \mathrm{BrN}\right)$ (Loba Chemie, India) were used without any further purification. Chemical structures of DTAB, TTBB and $\mathrm{CTAB}$ are shown in Fig. 1. Deionised water was used throughout the experiments. Ordinary Portland cement (OPC), 43 grade, conforming to IS: 8112 was used.

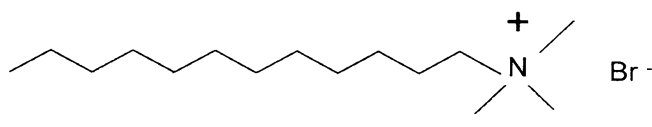

Dodecyl trimethyl ammonium bromide $\mathrm{C}_{15} \mathrm{H}_{34} \mathrm{NBr}$

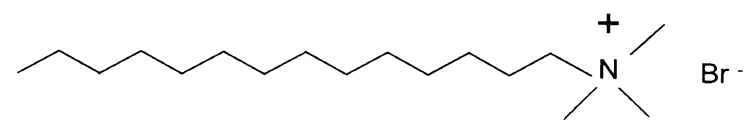

Tetradecyl trimethyl ammonium bromide $\mathrm{C}_{17} \mathrm{H}_{38} \mathrm{NBr}$

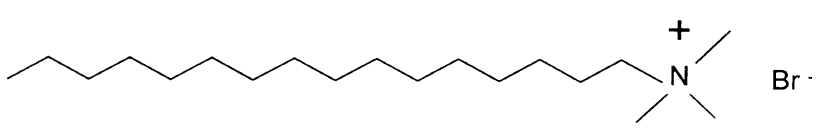

Cetyl trimethyl ammonium bromide $\mathrm{C}_{19} \mathrm{H}_{42} \mathrm{NBr}$

Fig. 1 Surfactant used in the synthesis process of silica nanoparticles

For each experiment, first ethanol, TEOS, water and surfactant were mixed and stirred for $30 \mathrm{~min}$. Then ammonia was added quickly to the previously prepared mixture. A white turbidity appears after addition of ammonia. The reaction was completed in $2 \mathrm{~h}$. The concentration of ammonia, water and TEOS were fixed at 0.2 , 3.2 and $0.2 \mathrm{M}$, respectively. The resulting white powder was dried overnight at $100^{\circ} \mathrm{C}$ and then calcined in air for a period of $3 \mathrm{~h}$ at $650^{\circ} \mathrm{C}$. The reaction was also performed without surfactants for comparative studies of particle size of silica nano particles.

Morphological attributes of nanosilica were measured using scanning electron microscope (LEO-438 VP) at an accelerating voltage of $20 \mathrm{kV}$. The samples were analyzed under high vacuum (HV) mode with gold coating so as to improve the image quality. The samples were deposited on a sample holder with a double stick conducting carbon tape. The average particle size was determined based on the measurement of the number of particles from the SEM micrographs. The term particle size used in this paper refers to the average diameter of the silica nano particles. Powder X-ray diffraction data were recorded at room temperature on a Rigaku D-Max 2200 using $\mathrm{Cu}-\mathrm{K} \alpha$ radiation at a scanning rate of $1 \mathrm{~min}^{-1}$. Surface modification of synthesized silica nanoparticles using cationic surfactants as organic templates was analyzed on a Bruker AV300 NMR spectrometer operating at $59.6 \mathrm{MHz}$ for ${ }^{29} \mathrm{Si}$. All NMR spectra were obtained using a contact time of $10 \mathrm{~ms}$ and a pulse repletion time of $0.5-1.0 \mathrm{~s}$. All chemical shifts are reported with respect to the silicon resonance in liquid $\mathrm{Me}_{4} \mathrm{Si}$. 


\section{Results and discussion}

The sol gel process involves the formation of a colloidal suspension (sol) and gelation of the sol to form a network in a continuous liquid phase (gel). Generally, tetraethoxysilane (TEOS) is used as a precursor and ammonia as catalyst for synthesizing nanosilica.

The chemical reaction of nanosilica synthesis can be summarized as:

Hydrolysis : $\mathrm{Si}-(\mathrm{OR})_{4}+4 \mathrm{H}_{2} \mathrm{O} \rightleftarrows \mathrm{Si}-(\mathrm{OH})_{4}+4 \mathrm{R}-\mathrm{OH}$

Condensation : $(\mathrm{OR})_{3}-\mathrm{Si}-\mathrm{OH}+\mathrm{HO}-\mathrm{Si}-(\mathrm{OR})_{3}$ $\rightarrow\left[(\mathrm{OR})_{3} \mathrm{Si}-\mathrm{O}-\mathrm{Si}(\mathrm{OR})_{3}\right]+\mathrm{H}-\mathrm{O}-\mathrm{H}$

There are a number of parameters that affect the process, including $\mathrm{pH}$, temperature, and concentration of reagents, $\mathrm{H}_{2} \mathrm{O}$ / $\mathrm{Si}$ molar ratio and other reaction conditions. When a silica source is combined with the head group of an cationic surfactant (CTAB) by either electrostatic force or hydrogen bond interaction in solution, the self-organization process is complicated, involving silicate speciation reactions, surfactant/ silicate self-assembly. Then the transformation of mesophases occurs during the hydrolysis and condensation of silica precursor. These processes are sensitive to concentration and type of surfactants, $\mathrm{pH}$, temperature, aging time, type, etc. In synthesis processes, there are at least three kinds of charged species in solution: silica species $\left(\mathrm{I}^{-}\right.$or $\left.\mathrm{I}^{+}\right)$, cationic surfactant $\mathrm{S}^{+}$and its counter ion $\left(\mathrm{X}^{-}\right)$. Under basic conditions, charged silica precursor and the surfactant of opposite charges form the strong interaction $\left(\mathrm{S}^{+}-\mathrm{I}^{-}\right.$or $\left.\mathrm{S}^{-}-\mathrm{I}^{+}\right)$in favor of highly ordered mesostructure. Once the negatively charged hydrolysis products are formed, they will slowly replace the bromides as counter ions of the cationic surfactants to electrostatically neutralize them, because bromide is known to be a strong binding anion. Moreover, the chosen reaction conditions of room temperature and the use of ammonia as the base promote a slowing of the hydrolysis of the TEOS molecules. The slow hydrolysis of TEOS forming a variety of silicate species in the solution, the disturbed mechanism of charge matching between the silicate species and the CTAB surfactant, the potential delayed condensation and slow polymerization of silicatesurfactant aggregates are all due to the massive presence of alcohol at the inorganic-organic interface. It all contributes to the complex mechanism, which provides the control growth of the micelles on the surfaces of the centre particles. The size and morphology of a growing particle also depends on the balance between the rate of polymerization of the negatively charged silicate micelles and the rate of mesostructure formation. In case of a slow polymerizing silicate seed in the presence of $\mathrm{CTAB}$, the growth is driven by the global surface tension forces to minimize its surface free energy by controlling the size of sphere at nano level, as observed by SEM. In the present study a series of cationic surfactants, tetradecyltrimethylammonium bromide, dodecytrimethylammonium bromide, cetyltrimethylammonium bromide were used to control the size and morphology of silica nano particles. Scanning electron microscopy (SEM) micrographs of spherical silica nanoparticles (Fig. 2) illustrated that the particles are spherical, well dispersed and possess a smooth surface morphology. Particle size could be controlled by varying the chain length of cationic surfactant. Particles produced using the aqueous TEOS precursor solution with cetyltrimethylammonium bromide displayed better dispercivity and lower size than those made with other two cationic surfactants. Silica particles in the range $150-50 \mathrm{~nm}$ could be prepared using a series of cationic surfactants, tetradecyltrimethylammonium bromide, dodecyltrimethylammonium bromide, cetyltrimethylammonium bromide. XRD pattern of silica nanoparticles is shown in Fig. 3. It can be concluded from the absence of peaks that the nanosilica synthesized by sol gel method is purely amorphous. A representative IR spectrum of nanosilica is shown in Fig. 4. The peaks can be identified as follows: (a) $\sim 472 \mathrm{~cm}^{-1}$ (Si-O rocking vibration), (b) $\sim 812 \mathrm{~cm}^{-1}$ ( $\mathrm{Si}-\mathrm{O}$ bending vibration), (c) $\sim 972 \mathrm{~cm}^{-1}$ ( $\mathrm{Si}-\mathrm{O}-\left(\mathrm{H} \cdots \mathrm{H}_{2} \mathrm{O}\right)$ bending vibration), (d) $\sim 1,076 \mathrm{~cm}^{-1}$ (asymmetric stretching vibration of $\mathrm{Si}-\mathrm{O}-\mathrm{Si}$ band), (e) $\sim 1,630 \mathrm{~cm}^{-1}$ (O-H bending vibration of adsorbed molecular water) and (f) $\sim 3,450 \mathrm{~cm}^{-1}$ ( $\mathrm{Si}-\mathrm{OH}$ stretching vibration, hydrogen bonded). Fig. 5 shows the ${ }^{29}$ Si MAS NMR spectra of all silica nanoparticles synthesized with and without cationic surfactants. The ${ }^{29} \mathrm{Si}$ MAS NMR spectra of silica nanoparticles show the $\mathrm{T}$ and $\mathrm{Q}$ sites. The signals at approx -113.0 and $-103.0 \mathrm{ppm}$ arise from the $\mathrm{Si}$ species $\mathrm{Q}^{4}$ $\left(\mathrm{Si}(\mathrm{OSi})_{4}\right)$ and $\mathrm{Q}^{3}\left(\mathrm{Si}(\mathrm{OH})(\mathrm{OSi})_{3}\right)$, respectively. A lowintensity peak at $-94.20 \mathrm{ppm}$ arises from chemical shift correlation and relaxation data to germinal-hydroxyl silanol sites (Wu et al. 2004).

The nanoparticles have high surface energy and atoms at the surface have a high activity, leading the atoms to react rapidly. The two silicate phases of cement, tricalcium silicate and dicalcium silicate, give calcium-silicate-hydrate (C-S-H) gel and calcium hydroxide $(\mathrm{CH})$ as hydration products, which constitute approximately $50-70 \%$ and $20-25 \%$ of the total volume of the hydrated product, respectively. The $\mathrm{C}-\mathrm{S}-\mathrm{H}$ gel being the main component of cement hydration is responsible for the strength and microstructure of the cement paste. XRD profiles of plain cement, silica nanoparticles and silica fume incorporated pastes at 28 days of hydration are shown in Fig. 6. It is evident from XRD profiles that the $\mathrm{CH}$ peak nearly disappeared with the addition of silica nanoparticles, while the same is significantly present in plain and silica fume incorporated cement paste. It is therefore inferred from Fig. 6 that addition of silica nanoparticles significantly consumes the $\mathrm{CH}$ produced during the hydration process. 

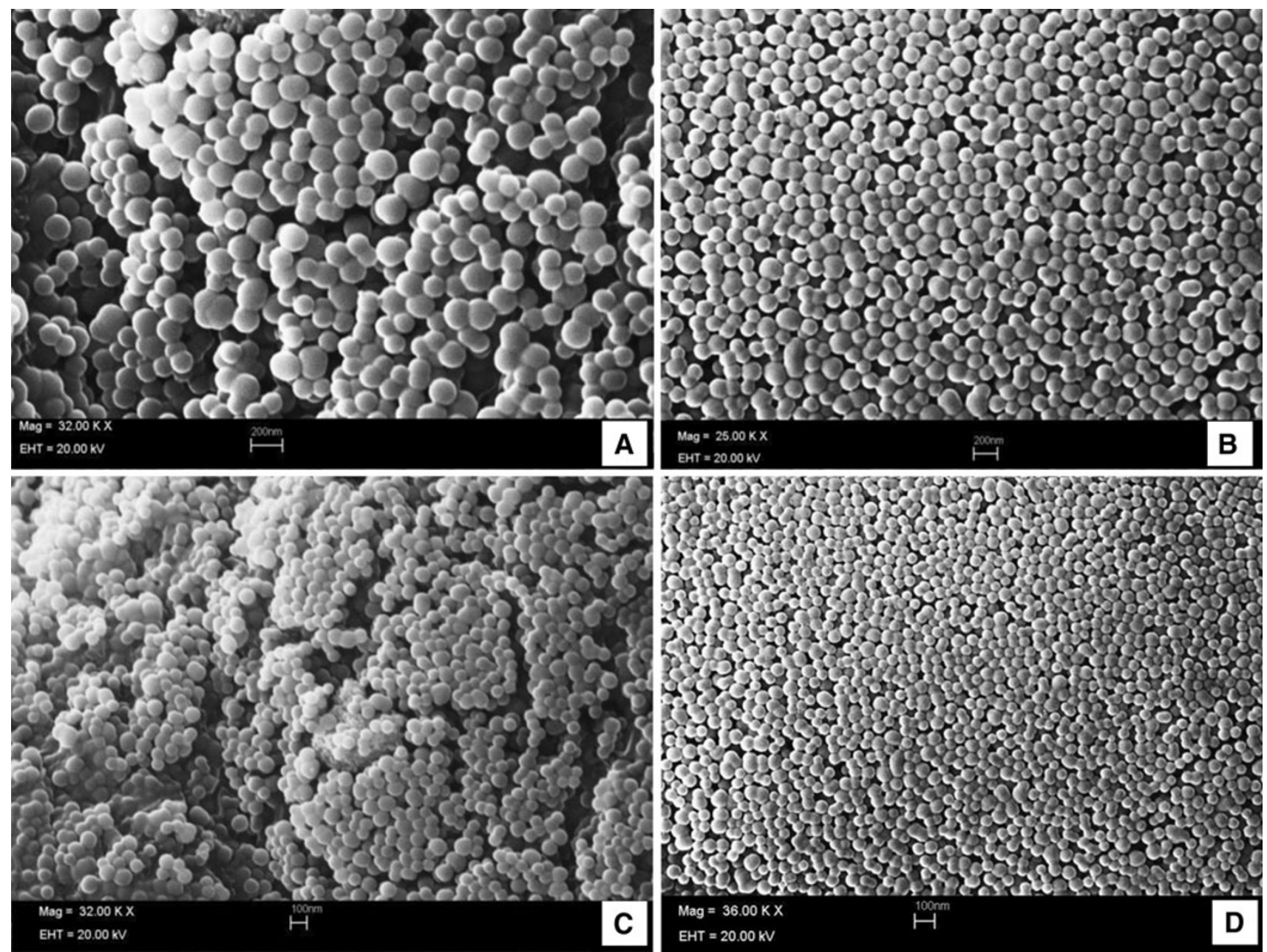

Fig. 2 SEM micrographs of silica nanoparticles synthesized without using surfactant (a) $(\sim 180 \mathrm{~nm})$ and using surfactants DTAB (b) $(\sim 140 \mathrm{~nm})$, TTAB $(\mathbf{c})(\sim 95 \mathrm{~nm})$ and CTAB $(\mathbf{d})(\sim 55 \mathrm{~nm})$

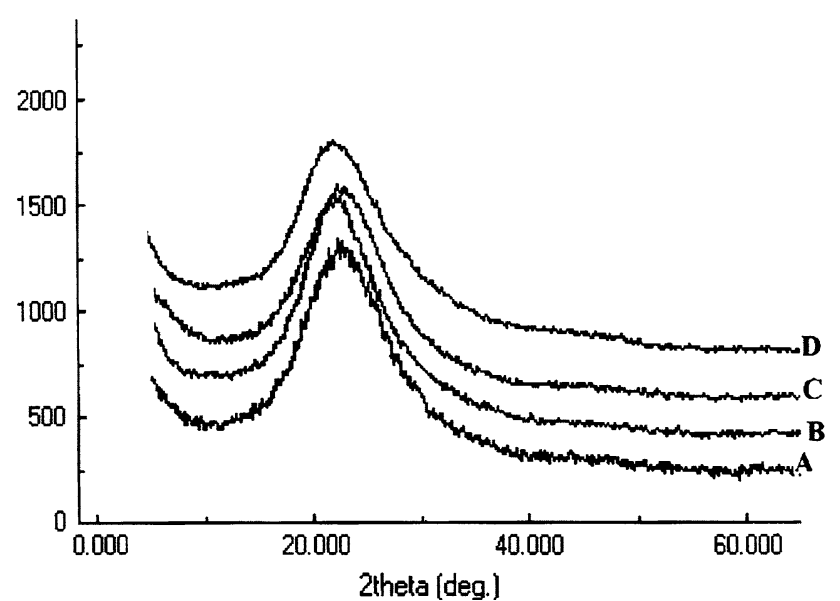

Fig. 3 XRD of silica nanoparticles synthesized by sol gel method, without surfactant $(A)$, with surfactants DTAB $(B)$, TTAB $(C)$ and CTAB $(D)$

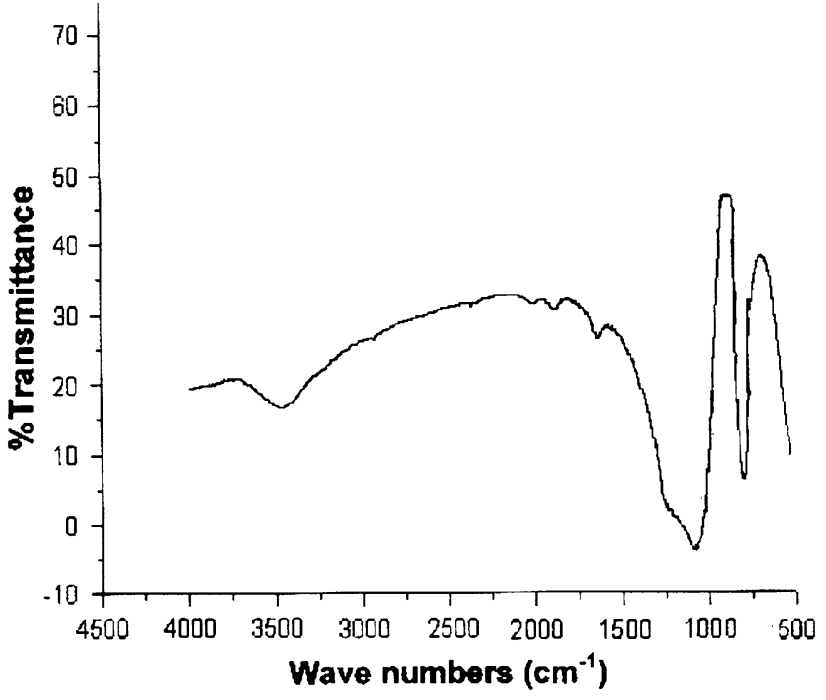

Fig. 4 IR spectra of synthesized silica nanoparticles 


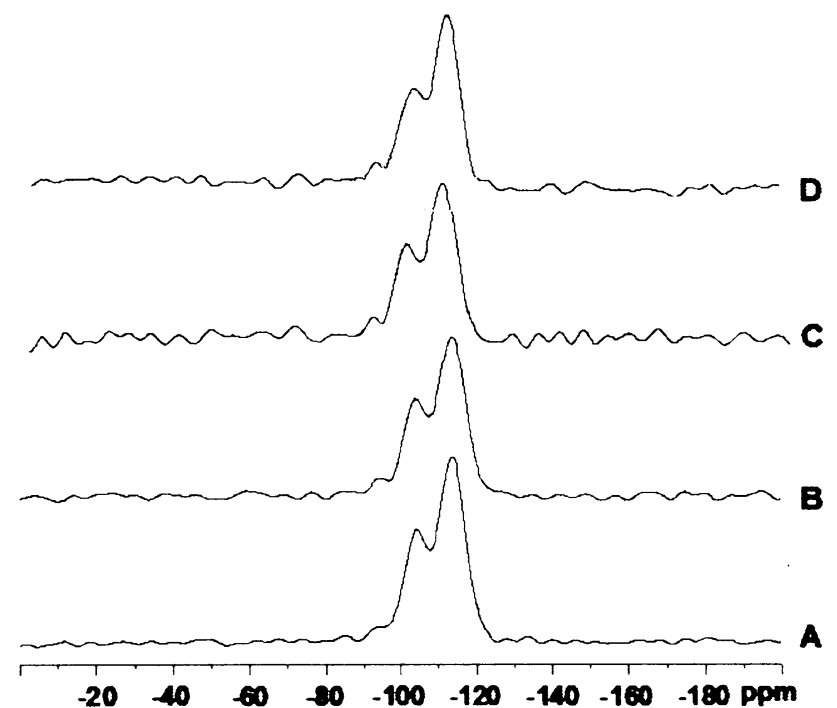

Fig. $5{ }^{29} \mathrm{Si}$ MAS NMR spectra of silica nanoparticles synthesized $A$ without surfactant, $B$ using DTAB, $C$ using TTAB and $D$ using CTAB

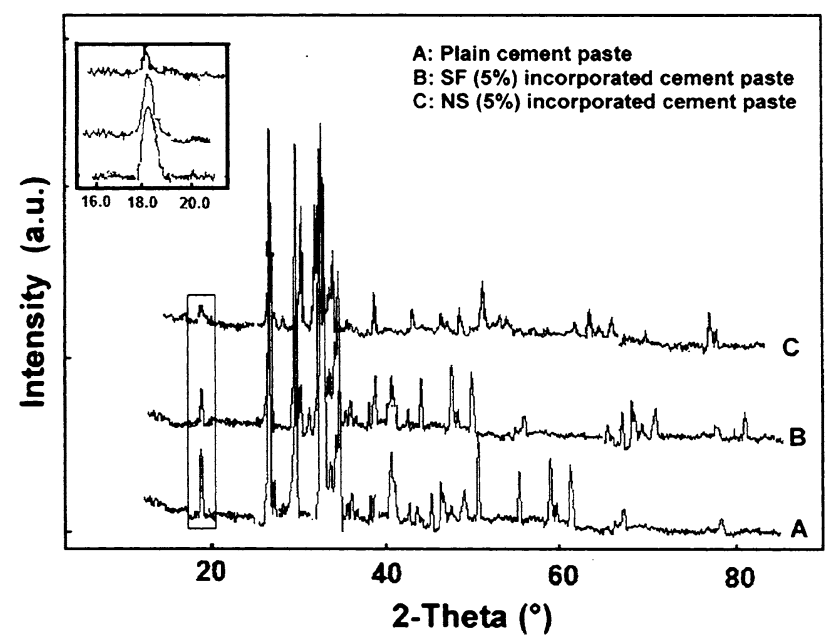

Fig. 6 XRD profiles of cement pastes at 28 days of hydration

Therefore, the pozzolanic reactivity of silica nanoparticles at early stage of hydration is significantly high and increases compressive strength at early stages, thereby enhancing the durability and mechanical properties of the cementitious materials.

\section{Conclusions}

Amorphous, spherical and uniform size silica nano particles can be prepared by the hydrolysis reaction of TEOS as a silica precursor in ethanol and ammonia as a catalyst using sol gel method. The particle size of silica nanoparticles can be controlled using dodecytrimethylammoniumbromide $(\sim 140 \mathrm{~nm})$, tetradecyltrimethylammoniumbromide $(\sim 95 \mathrm{~nm})$, cetyltrimethylammonium bromide $(\sim 55 \mathrm{~nm})$ as cationic surfactants. The particle size decreases as the chain length of the surfactants is increased. Pozzolanic reaction induced by silica nanoparticles prevents early calcium leaching as it digested $\mathrm{CH}$ crystals and improved the microstructure by forming denser $\mathrm{C}-\mathrm{S}-\mathrm{H}$ gel. During the hydration of cement, silica nanoparticles can fill the pores and voids effectively due to their ultrafine size and high pozzolanic activity and thus starting the act as reaction nucleus. Thus, addition of silica nanoparticles accelerates the hydration of cement at early stages.

Acknowledgments One of the authors (S. Ahalawat) is grateful to UCOST, Dehradun for fellowship assistance.

Open Access This article is distributed under the terms of the Creative Commons Attribution License which permits any use, distribution and reproduction in any medium, provided the original author(s) and source are credited.

\section{References}

Awaji N, Ohkubo S, Nakanishi T, Aoyama T, Sugita Y, Takasaki K, Komia S (1997) Thermal oxide growth at chemical vapor deposited $\mathrm{SiO}_{2} / \mathrm{Si}$ interface during annealing evaluated by difference X-ray reflectivity. Appl Phys Lett 71:1954

Bore MT, Rathod SB, Ward TL, Datye AK (2003) Hexagonal mesostructure in powders produced by evaporation-induced selfassembly of aerosols from aqueous tetraethoxysilane solutions. Langmuir 19:256-264

Grün M, Unger KK, Matsumoto A, Tsutsumi K (1999) Novel pathways for the preparation of mesoporous MCM-41 materials: control of porosity and morphology. Microporous Mesoporous Mater 27:207-216

Hong RY, Feng B, Ren ZQ, Xu B, Li HZ, Zheng Y (2009) Thermodynamic, hydrodynamic, particle dynamic, and experimental analyses of silica nanoparticles synthesis in diffusion flame. Can J Chem Eng 87:143-156

Lebedev OI, Tendeloo GV, Collart, Cool O, Vansant PEF (2004) Structure and microstructure of nanoscale mesoporous silica spheres. Solid State Sci 6:489-498

Liu S, Cool P, Collart O, Voort PVD, Vansant EF, Lebedev OI, Tendeloo GV, Jaing M (2003) The influence of the alcohol concentration on the structural ordering of mesoporous silica:cosurfactant versus cosolvent. J Phys Chem B 107:10405

Miller CR, Vogel R, Surawski PPT, Jack KS, Corrie SR, Trau M (2005) Functionalized organosilica microspheres via a novel emulsion-based route. Langmuir 21:9733

Oh C, Lee YG, Park JH, Oh SG (2005) Synthesis of silica microspheroids for templates in W/O reverse emulsion. Colloids Surf A 269:112-118

Pauwels B, Tendeloo GV, Thoelen C, Rhijn WV, Jacobs PA (2001) Structure determination of spherical MCM-41 particles. Adv Mater 13:1317-1320

Rao GVR, López GP, Bravo J, Pham H, Datye AK, Xu H, Ward TL (2002) Monodisperse mesoporous silica microspheres formed by evaporation-induced self assembly of surfactant templates in aerosols. Adv Mater 14:1301-1304 
Shimura N, Ogava M (2005) Growth of nanoporous silica spherical particles by the stöber method combined with supramolecular templating approach. Bull Chem Soc Jpn 78:1154-1159

Tan B, Rankin SE (2004) Interfacial alignment mechanism of forming spherical silica with radially oriented nanopores. J Phys Chem B 108:20122

Tan B, Lehmler HJ, Vyas SM, Knuston BL, Rankin SE (2005) Controlling nanopore size and shape by fluorosurfactant templating of silica. Chem Mater 17:916-925

Venkatathri N (2007) Synthesis of mesoporous silica nanosphere using different templates. Solid State Commun 143:493-497
Venkatathri N, Nanjundan S (2009) Synthesis and characterization of a mesoporous silica microsphere from polystyrene. Mater Chem Phys 113:933-936

Wu D, Zhai SR, Gong YJ, Zhang Y, Deng F, Luo Q, Sun YH (2004) Mixed cationic-nonionic surfactants and $\mathrm{pH}$ adjustment route to synthesize high-quality MCM-48. J Chin Chem Soc 51:49-57

Yongqi H, Zhao H, Li Y (2008) Synthesis method for silica needleshaped nano-hollow structure. Mater Lett 62:3401-3403

Zhang YB, Qian XF, Li ZK, Yin J, Zhu ZK (2004) Synthesis of novel mesoporous silica spheres with starburst pore canal structure. J Solid State Chem 177:844-848 